\title{
Zukunft der deutschen Energiegenossenschaften
}

\author{
Herausforderungen und Chancen aus einer Innovationsperspektive
}

\author{
Britta Klagge $^{1} \cdot$ Hanna Schmole $^{2} \cdot$ Irmi Seidl $^{3} \cdot$ Susanne Schön $^{4}$
}

Eingegangen: 5. Januar 2016 / Angenommen: 11. Mai 2016 / Online publiziert: 6. Juni 2016

(C) Springer-Verlag Berlin Heidelberg 2016

Zusammenfassung Energiegenossenschaften haben für den dezentralen Ausbau der erneuerbaren Stromproduktion in Deutschland im neuen Jahrtausend eine wichtige Rolle gespielt: Sie bündeln das Kapital von Privatpersonen und erschließen damit eine weitere Finanzierungsquelle für das Erreichen der Energiewende. Dabei gelten ihre regionale Orientierung und die genossenschaftliche Mitbestimmung als akzeptanzfördernde Erfolgsfaktoren. Mit der Novellierung des Erneuerbare-Energien-Gesetzes (EEG) 2014 fallen nun die bisher sehr günstigen Förderbedingungen sukzessive weg, die für die Geschäftsmodelle vieler, vor allem regionaler Energiegenossenschaften zentral waren. Damit ist die Boomphase der Energiegenossenschaften beendet und die zunehmende Marktorientierung der Förderpolitik für erneuerbare Energien erfordert von den Energiegenossenschaften Anpassungen und Innovationen. Vor diesem Hintergrund werden Energiegenossenschaften und ihre Entwicklung aus einer Innovationsperspektive im Hinblick darauf untersucht, wie sich Energiegenossenschaften positionieren müssen, wenn sie auch in Zukunft einen bedeutsamen Beitrag zu einer dezentralen Energiewende leisten wollen bzw. sollen.

Britta Klagge

klagge@uni-bonn.de

1 Geographisches Institut der Universität Bonn, Meckenheimer Allee 166, 53115 Bonn, Deutschland

2 EuPD Research, Adenauerallee 134, 53113 Bonn, Deutschland

3 Swiss Federal Research Institute WSL, Zürcherstrasse 111, 8903 Birmensdorf, Schweiz

4 inter 3 Institut für Ressourcenmanagement, Otto-Suhr-Allee 59, 10585 Berlin, Deutschland
Im empirischen Teil dieses Beitrags werden auf der Basis vorhandener Daten, einer eigenen Online-Befragung mit einem Schwerpunkt auf Energiegenossenschaften unterschiedlicher Größe und Reichweite sowie ergänzt durch zwei Fallstudien die Strukturen und der Wandel im deutschen Energiegenossenschaftssektor analysiert. Wir schlussfolgern, dass Größenvorteile und Risikostreuung durch überregionale Aktivität zwar Vorteile bieten können, dass es daneben aber auch für regionale Energiegenossenschaften weitere erfolgversprechende Entwicklungsmöglichkeiten gibt. Ansatzpunkte für erfolgreiche regionale Strategien bilden die Diversifikation der Geschäftsfelder, die neben Energiedienstleistungen auch weitere Tätigkeiten der örtlichen Daseinsvorsorge beinhalten können, sowie Kooperationen auf regionaler Ebene und mit anderen Energiegenossenschaften bzw. deren Dachverbänden. Generell bildet die Direktvermarktung ein potenziell relevantes Element der innovativen Weiterentwicklung von Geschäftsmodellen, da sie - entsprechend den genossenschaftlichen Kernprinzipien Mitgliederorientierung und Identitätsprinzip - die Fokussierung auf bestimmte Zielgruppen mit der Vermittlung eines regionalen bzw. ideellen Mehrwerts verknüpft.

Schlüsselwörter Energiegenossenschaften · Erneuerbare Energien · Innovation · EEG-Novellierung · Deutschland

\section{Future of German energy co-operatives}

Challenges and opportunities in an innovation perspective

Abstract Energy co-operatives have played an important role in the development of decentralized renewable electricity production in Germany in the new millennium. They collect capital from individuals and thus open up another 
source of funding to promote the "Energiewende" in Germany. Their regional orientation and the co-operative participation model are regarded as success factors which help to increase local acceptance. With the amendment of the German Renewable Energy Sources Act (EEG) in 2014, the so far very favorable funding conditions gradually fall away, which were central to the business models of many, especially regional energy co-operatives. The boom phase of energy co-operatives has thus come to an end and the increasing market orientation of support policies requires adaptations and innovations. Against this background the paper explores energy cooperatives and their development from an innovation perspective. It specifically asks how they need to position, if they are to make a significant contribution to a decentralized energy transition in the future.

In the empirical part of this paper, we examine the structures and developments in the German energy co-operative sector based on existing data and an online survey, with a focus on energy cooperatives of different sizes and geographical orientation, complemented by an analysis of two case studies. We conclude that economies of scale and geographical diversification can offer benefits, but that there are also promising development opportunities for regional energy co-operatives. Diversification of business fields, which can include not only energy-related services, but also other local general interest services, as well as cooperation at the regional level and with other energy co-operatives and their umbrella associations, could be starting points for successful regional strategies. In general, direct marketing can become a potentially important element of innovative development of business models, because - in accordance with the co-operative core principles of member orientation and the principle of identity - it links the focus on specific target groups with a regional or ideological surplus value.

Keywords Energy cooperatives - Renewable energies · Innovation · EEG amendment · Germany

\section{Einleitung}

Die Energiewende in Deutschland ist innerhalb weniger Jahre insbesondere im Bereich der Stromerzeugung deutlich vorangeschritten; so wurde im Jahr 2015 bereits rund ein Drittel des verbrauchten Stroms aus erneuerbaren Energien (EE) gewonnen. Energiegenossenschaften (EG) haben insbesondere für den dezentralen Ausbau der Stromproduktion eine wichtige Rolle gespielt (DGRV 2013: 3; Engerer 2014: 1; Klemisch 2014a: 22). Sie bündeln das Kapital von Privatpersonen und erschließen damit eine Finanzierungsquelle jenseits des Finanz- und Unternehmenssektors. Als überwiegend regional operierende und demokratisch verfasste Organisationen können sie zudem zu einer besseren
Koordination regionaler EE-Maßnahmen sowie gleichzeitig zur lokalen Wertschöpfung und kommunalen Einnahmenerzielung beitragen - und damit die Akzeptanz für die Energiewende insgesamt erhöhen (George 2012: 506; Jakubowski/Koch 2012: 489; Volz 2012a; Klemisch 2014a: 22). Vor diesem Hintergrund ist die Institution Energiegenossenschaft für die raumwissenschaftliche Forschung zur Energiewende von besonderem Interesse.

Zweifellos wurden Energiegenossenschaften in den letzten Jahren vor allem durch das Erneuerbare-Energien-Gesetz (EEG) und die garantierten Einspeisevergütungen befördert; sie stellen eine bedeutende soziale Innovation bei der Umsetzung der Energiewende dar. Mit der Novellierung des EEG von 2014 fallen wesentliche begünstigende Rahmenbedingungen sukzessive weg, was bereits $\mathrm{zu}$ einem deutlichen Rückgang der Neugründungs- und der Investitionstätigkeit geführt hat (DGRV 2014: 5; Müller/ Holstenkamp 2015). Auf diese Veränderungen muss der Energiegenossenschaftssektor mit Anpassungen und neuen Geschäftsmodellen reagieren. Welche Bedeutung können Energiegenossenschaften im neuen Energiemarktdesign haben und wie müssen sie sich unter den neuen Rahmenbedingungen positionieren, um weiterhin eine energiewirtschaftliche und -politische Bedeutung haben zu können?

Im vorliegenden Beitrag wird die Rolle von Energiegenossenschaften angesichts einer zunehmenden Marktorientierung der Förderpolitik in Bezug auf erneuerbare Energien analysiert. Untersuchungsleitend ist dabei folgende Hypothese: Mit dem Fortschreiten der Energiewende sowie neuen institutionellen Bedingungen müssen sich die Strukturen und die Reichweite von Energiegenossenschaften verändern, wenn diese auch in Zukunft einen signifikanten Beitrag zu einer Energiewende leisten sollen, die auf dezentrale Strukturen der Erzeugung und des Verbrauchs von Energie orientiert ist. Insbesondere eine überwiegend regionale Orientierung und damit häufig einhergehend eine geringe Größe könnten der erfolgreichen Fortsetzung der genossenschaftlichen Finanzierung der Energiewende entgegenstehen - obwohl sie gleichzeitig Teil ihres bisherigen Erfolgsrezeptes waren. Damit fokussiert der Beitrag auf die Bedeutung von Größe und regionaler Orientierung für zukünftige Entwicklungsperspektiven von Energiegenossenschaften.

Im folgenden zweiten Abschnitt werden zunächst im Kontext des Genossenschaftsgedankens Grundlagen zu Energiegenossenschaften, ihren Erfolgsfaktoren und aktuellen Herausforderungen beschrieben; außerdem wird der Ansatz der Innovationsbiographie eingeführt, seine Anwendung im vorliegenden Zusammenhang begründet und darauf basierend in die Fragestellung eingeleitet. Der dritte Abschnitt widmet sich den aktuellen Strukturen und dem Wandel im deutschen Energiegenossenschaftssektor, wobei vertieft auf die Faktoren Größe und regionale Ori- 
entierung eingegangen wird. Die skizzierte Entwicklung des Energiegenossenschaftssektors wird dann mit dem innovationsbiographischen Ansatz interpretiert. Im vierten Abschnitt wird zwischen regionalen und überregionalen Energiegenossenschaften differenziert und deren Unterschiede in Bezug auf Tätigkeitsfelder und Kooperationen analysiert; zusätzlich werden zwei Fallbeispiele vorgestellt. Der fünfte und letzte Abschnitt fasst die Ergebnisse zusammen und diskutiert Ansatzpunkte erfolgversprechender Entwicklungsmöglichkeiten für überregionale und regionale Energiegenossenschaften. Die empirischen Analysen basieren auf Daten und anderen Informationen aus der Literatur sowie zudem auf den Ergebnissen einer Anfang 2014 an der Universität Bonn durchgeführten Online-Befragung von Energiegenossenschaften.

\section{Rechtliche Hintergründe, Erfolgsfaktoren und Herausforderungen: innovationstheoretische Überlegungen}

Der Erfolg von Energiegenossenschaften in der deutschen Energiewende hängt eng mit ihrer Rechtsform zusammen, die finanzielle Beteiligung mit demokratischer Mitbestimmung verknüpft und damit eine umfassende Form der Bürgerbeteiligung ermöglicht (2.1). Neben dieser starken Form der Mitbestimmung gelten die damit häufig verbundene regionale Orientierung sowie die - bisher im Strombereich allerdings eher seltene - Verbindung von Produktion und Konsumtion als Merkmale von Energiegenossenschaften, die die Akzeptanz der Energiewende vor Ort erhöhen (2.2). Die Neuregelung des EEG im Jahr 2014 gefährdet jedoch das bisher überwiegend verfolgte Geschäftsmodell und damit die Tätigkeit sowie das Wachstum des (energie-)genossenschaftlichen Sektors (2.3), was die Frage nach einer stärkeren Professionalisierung aufwirft (2.4). Aus einer innovationsbiographischen Perspektive stellt sich dies als Bruch dar, als ein Entwicklungsknick, der eine neue Phase einleitet und die Frage nach den zukünftigen Entwicklungsmöglichkeiten aufwirft (2.5).

\subsection{Genossenschaften als demokratische Rechtsform gemeinschaftlicher Organisation und Finanzierung}

In der umfangreichen Literatur zum Thema Genossenschaften besteht Einigkeit über drei Kernprinzipien, die die Institution Genossenschaft von anderen unternehmerischen Organisationsformen unterscheiden (z. B. Volz 2011; Henkel 2013): (1) Mitgliederorientierung, das heißt, Genossenschaften verfolgen wirtschaftliche, soziale oder kulturelle Belange ihrer Mitglieder durch gemeinschaftlichen Geschäftsbetrieb; (2) Identitätsprinzip, das besagt, dass zwei normalerweise gegenüberstehende Rollen zusam- menfallen, z. B. Produzent und Konsument, Kapitaleigner und Beschäftigter, Vermieter und Mieter etc., wobei viele Energiegenossenschaften eine Ausnahme bilden, da sie Energie produzieren, aber die Mitglieder diese Energie nicht selbst konsumieren; (3) Demokratieprinzip, das bestimmt, dass jedes Genossenschaftsmitglied eine Stimme hat, unabhängig von der Zahl der Anteile bzw. der Höhe der Kapitaleinlage. Weitere zentrale Merkmale oder Prinzipien sind die freiwillige und offene Mitgliedschaft, die Zusammenarbeit mit anderen Genossenschaften sowie die Berücksichtigung gemeinschaftlicher Ziele bzw. des Gemeinwohls: „Co-operatives are businesses owned and run by and for their members. [...] [As they are] driven by values not just profit, [...] [they] share internationally agreed principles and act together to build a better world through co-operation“ (ICA 2011).

Damit ermöglichen eingetragene Genossenschaften $(\mathrm{eG})$ die unmittelbarste und demokratischste Form gemeinschaftlicher Finanzierung ${ }^{1}$, nicht nur im Energiesektor. In der Regel sind Energiegenossenschaften im (überwiegenden) Eigentum von Bürgern und werden maßgeblich von ihnen gesteuert - besonders oft zusammen mit Gemeinden, aber auch mit anderen öffentlichen, privaten oder zivilgesellschaftlichen Akteuren; hinzu kommt häufig eine regionale Orientierung (George 2012: 506; DGRV 2014: 15). Daher stehen Genossenschaften für eine starke Form der Bürgerbeteiligung in der Energiewende. Hierbei ist zu berücksichtigen, dass sich der deutsche Begriff Bürgerbeteiligung sowohl auf die finanzielle Beteiligung, also die Bereitstellung von Kapital, als auch auf die Mitbestimmung beziehen kann. Die Autoren der Studie „Definition und Marktanalyse von Bürgerenergie in Deutschland" Degenhart und Holstenkamp verstehen dies als „Bürgerenergie im engeren Sinne“ und meinen damit überwiegend regionale Unternehmen, in denen mindestens 50 Prozent der Stimmrechte bei Bürgern aus der Region liegen (Trend:research/Leuphana Universität Lüneburg 2013: 28-29).

\subsection{Regionale Orientierung und Mitbestimmung als Erfolgsfaktoren von Energiegenossenschaften}

Der bisherige Erfolg von Energiegenossenschaften ist eng mit ihrer regionalen Orientierung verbunden (Schröder/ Walk 2013; Schröder/Walk 2014; Klemisch 2014a). Damit „passen“ Energiegenossenschaften zu den erneuerbaren Energien, denn deren Nutzung weist eine deutlich dezentralere Organisations- und Raumstruktur auf als die fossile

\footnotetext{
1 Weitere Rechtsformen, die zur gemeinschaftlichen Finanzierung von Erneuerbare-Energie-Projekten genutzt werden, sind Vereine (e. V.), GmbH \& Co. KGs, GmbHs, Kommanditgesellschaften (KGs), Gesellschaften bürgerlichen Rechts (GbRs) und manchmal sogar Aktiengesellschaften (AGs); für einen Überblick siehe Staab 2013.
} 
und nukleare Energieerzeugung (Brücher 2009; Klagge/ Brocke 2012) und sie eröffnen vielen Regionen lokale Wertschöpfungspotenziale (Hirschl/Aretz/Prahl et al. 2010; Flieger 2011). Die Entwicklung in Deutschland zeigt, dass insbesondere ländliche und periphere Räume wichtige Energiestandorte geworden sind und diese Regionen von verschiedenen, auf erneuerbare Energien bezogenen Maßnahmen profitieren, nicht nur wirtschaftlich, sondern auch im Hinblick auf regionale Identität und Selbstbestimmung. Letzteres ist vor allem dann gegeben, wenn regionale Akteure an den Planungs-, Entwicklungs- und Steuerungsprozessen sowie nicht zuletzt an der Finanzierung von Erneuerbare-Energien-Projekten beteiligt sind (Ribi/Buser/von Felten et al. 2012; Klagge/Brocke 2012; Klagge/Brocke 2013; Li/Birmele/Schaich et al. 2013).

Studien zur Bürgerbeteiligung an Erneuerbare-EnergienProjekten bestätigen, dass deren Akzeptanz sowie die entsprechende politische Unterstützung mit der Beteiligung an den Entscheidungsprozessen steigen (Walker 2008; Itten/ Mono 2014). Insbesondere regional operierende Energiegenossenschaften sind besser als externe Akteure in der Lage, Chancen und Hemmnisse auf der regionalen Ebene zu erkennen und in ihren Strategien zu berücksichtigen, beispielsweise indem sie durch Kooperation mit weiteren Akteuren vor Ort Koalitionen bilden, denen es gelingt, lokale Widerstände zu überwinden. Regionalökonomische Effekte, also z. B. Arbeitsplätze vor Ort, sowie finanzielle Vorteile für die Kommune oder sogar beteiligte Individuen, insbesondere in Form von Steuereinnahmen und Einkommen aus Dividenden, sind zusätzliche Faktoren, die die Unterstützung für erneuerbare Energien erhöhen und NIMBY-(not in my backyard)-Einstellungen (die auf dem „Sankt-FloriansPrinzip“ beruhen) etwas entgegensetzen können (Schreuer/Weismeier-Sammer 2010; Zoellner/Rau/Schweizer-Ries 2011: 26; Li/Birmele/Schaich et al. 2013; Masson/Centgraf/ Rauschmayer et al. 2015; für einen Überblick und eine kritische Bewertung der Literatur zu Akzeptanzfragen siehe auch Cowell/Bristowa/Munday 2011).

Interessant ist, dass die im Genossenschaftsgedanken angelegte Einheit von Produktion und Konsumtion (Identitätsprinzip, vgl. Abschnitt 2.1) insbesondere in stromerzeugenden Energiegenossenschaften bisher eher selten realisiert wird, obwohl gerade diese, vor allem in regionalen Kontexten, ein bedeutender akzeptanzfördernder Faktor sein könnte (Fischer/Moser/Schenk 2015). So waren im Jahr 2013 nur rund 10 Prozent der Energiegenossenschaften im Geschäftsfeld der regionalen Direktvermarktung aktiv. Dies hängt mit der Spezifität des Gutes „Energie“ bzw. Strom (z. B. seiner schlechten Speicherbarkeit) wie auch mit der Ausgestaltung des Erneuerbare-Energien-Gesetzes zusammen, das die Stromabnahme durch das (anonyme) Netz garantiert. Angesichts der Änderungen im EEG (vgl. Abschnitt 2.3) könnte sich dies jedoch ändern. Immerhin dach- ten 2013 bereits mehr als die Hälfte (52\%) der Energiegenossenschaften über die regionale Direktvermarktung nach (DGRV 2013: 18).

Bedeutsamer als die Einheit von Produktion und Konsumtion dagegen ist das Prinzip der Gemeinwohlorientierung. Neben den bereits genannten ökonomischen bzw. finanziellen Effekten vor Ort gibt es vereinzelt auch Genossenschaften, die den lokalen Kindergarten oder Entwicklungshilfeprojekte im globalen Süden unterstützen (vgl. Lasch 2009; Flieger 2011: 53). ${ }^{2} \mathrm{Zu}$ den Motiven für eine (mögliche) Beteiligung an Energiegenossenschaften und insbesondere zur Renditeorientierung für kollektiv finanzierte Erneuerbare-Energien-Projekte liegen einige Ergebnisse aus Fallstudien und Befragungen vor. So kann der individuelle finanzielle Nutzen für einige Investoren und Genossenschaftsmitglieder große Bedeutung haben (siehe z. B. Schreuer/Weismeier-Sammer 2010 zu BürgerWindparks, die allerdings in der Regel nicht als Genossenschaft organisiert sind). Andere Studien zeigen jedoch, dass unter Energiegenossenschafts-Mitgliedern und -Interessierten die Bedeutung nichtmaterieller Ziele überwiegt (Volz 2011; Volz 2012b; DGRV 2012: 14; Trend:research/ Leuphana Universität Lüneburg 2013; Radtke 2014: 7; Masson/Centgraf/Rauschmayer et al. 2015: 203).

\subsection{Aktuelle Herausforderungen durch die Neuregelungen des EEG im Jahr 2014}

Das im neuen Erneuerbare-Energien-Gesetz von 2014 (EEG 2.0) angelegte Marktdesign hat weitreichende Auswirkungen auf Art und Organisation der zukünftigen Tätigkeiten von Energiegenossenschaften. Dies betrifft vor allem das unter Energiegenossenschaften weit verbreitete Geschäftsmodell, das lediglich auf Aktivitäten in der Stromerzeugung basiert und bei dem Energiegenossenschaften den erzeugten Strom in das Netz einspeisen und gemäß EEG vergüten lassen (DGRV 2014: 9). Mit der für die Zukunft geplanten Ausweitung des Auktionsmodells von zunächst nur Fotovoltaik-Freiflächen auf andere Technologien, der Verpflichtung zur Direktvermarktung für alle neuen Anlagen ab $100 \mathrm{~kW}$ Leistung (ab 2016) und damit verbunden der sukzessiven Abschaffung der garantierten Einspeisevergütungen wird dem bisherigen Geschäftsmodell die Grundlage entzogen. Diese und weitere Veränderungen wie die Ausweitung der Erhebung der EEG-Umlage auf Eigenverbrauch und der Wegfall des Grünstromprivilegs werden von Vertretern und Befürwortern von Energiegenossenschaften sowie generell einer dezentralen Energiewende mit dem Argument kritisiert, dass sie kleine und weniger finanzstarke Akteure mit ei-

\footnotetext{
2 Vergleiche für weitere Beispiele etwa auch die Darstellungen unter www.öeg-horb.de oder www.fairpla.net.
} 
Abb. 1 Entwicklung der Neugründungen von Energiegenossenschaften und der Neueintragungen in das Genossenschaftsregister, 2006-2014 (Zwischen dem Gründungsdatum und der Eintragung in das Genossenschaftsregister können mehrere Monate liegen, da für die Eintragung eine Gründungsprüfung erforderlich ist.) (Quellen: DGRV 2014; Müller/Holstenkamp 2015; eigene Darstellung)

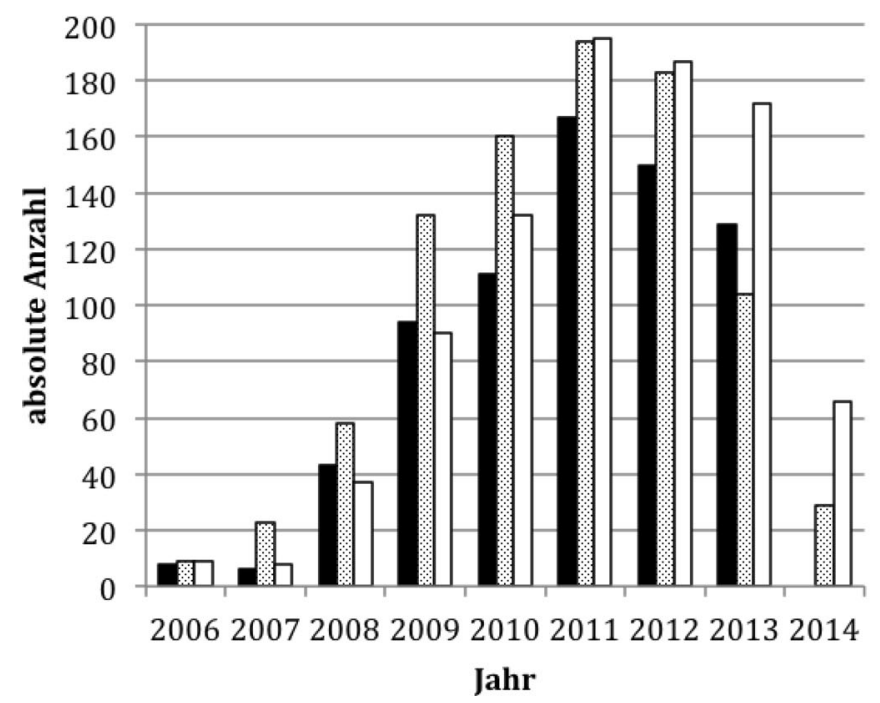

- Neugründungen gemäß DGRV (2014)

웅 Neugründungen gemäß Müller/ Holstenkamp (2015)

$\square$ Neueintragungen gemäß Müller/ Holstenkamp (2015) ner regionalen Orientierung benachteiligen (EnGeno 2014; BBEn 2014; Maly/Meister/Schomerus 2014; Engerer 2014: 4).

Ein Argument ist, dass Energiegenossenschaften häufig zu klein bzw. aufgrund ihrer regionalen Orientierung nicht in der Lage sind, die z. B. mit Ausschreibungsverfahren verbundenen Risiken und Transaktionskosten zu tragen, ohne ihre wirtschaftliche Grundlage zu gefährden (Degenhart/ Nestle 2014: viii). Insbesondere können sie die Projektrisiken nicht auf mehrere Projekte verteilen, sodass für den Fall, dass sie bei einer Ausschreibung nicht den Zuschlag erhalten, dies aufgrund des hohen Aufwands für die Teilnahme und der langen Planungsvorlaufzeiten existenzgefährdend sein kann. Konkrete Probleme, die sich aus der EEG-Novelle von 2014 für Energiegenossenschaften ergeben, sind damit steigende Finanzierungs- und Transaktionskosten, eine zunehmende Abhängigkeit von anderen Akteuren sowie damit verbunden ein Anstieg der finanziellen Risiken. Die hieraus resultierende Verunsicherung wird unter anderem durch einen Rückgang der Investitionen und einen Rückgang bei Neugründungen von Energiegenossenschaften illustriert; konkret spricht der Deutsche Genossenschafts- und Raiffeisenverband e. V. (DGRV) davon, dass allein 2014 Investitionen in Höhe von schätzungsweise 300 Millionen Euro zurückgestellt worden seien (DGRV 2014: 5; Müller/Holstenkamp 2015) (siehe Abb. 1). Ergänzungen bzw. Gegenvorschläge zur Novelle zielen darauf $a b$, kleinen Anbietern weiterhin eine Chance zu geben, beispielsweise durch exklusive Ausschreibungsrunden für kleine und mittlere Unternehmen (KMU) oder die Begrenzung der Risiken im Bieterverfahren. Unabhängig vom Erfolg dieser Vorschläge werden die veränderten Bedingungen die zukünftige Geschäftstätigkeit und Ausrichtung von Energiegenossenschaften erheblich beeinflussen.

\subsection{Professionalisierung von Energiegenossenschaften als mögliche Reaktion}

Zwar zielt die EEG-Novelle nicht auf eine Neuordnung des Energiegenossenschaftssektors, beeinträchtigt aber in erheblichem Maße die Geschäftstätigkeit und die Wirtschaftlichkeit von Energiegenossenschaften. Damit Energiegenossenschaften auch zukünftig in der Lage sind, einen spürbaren Beitrag zur (dezentralen) Finanzierung des Ausbaus erneuerbarer Energien zu leisten, müssen sie die bisher verfolgten Geschäftsmodelle weiter entwickeln und sich im Rahmen des genossenschaftlichen Ansatzes professionalisieren (Radtke 2013: 174; EnGeno 2014: 5, 8 f.; Klemisch 2014a: 23).

Die Professionalisierung kann mehrere Dimensionen umfassen. Maßgeblich ist das Realisieren von Größenvorteilen, wobei dies einerseits Größenwachstum der (einzelnen) Genossenschaften und andererseits die Entwicklung neuer Kooperationsformen und -strukturen umfassen kann. Letztere können Kooperationen zwischen Energiegenossenschaften (z. B. im Rahmen von Dachorganisationen) sein oder aber die Zusammenarbeit mit weiteren Akteuren wie Stadtwerken, Kommunen und Wohnungsbaugenossenschaften beinhalten (EnGeno 2014: 8 f.; Klemisch 2014a: 23).

Eng damit verbunden ist die Diskussion über die Diversifizierung der Tätigkeitsfelder von Energiegenossenschaften und damit die Entwicklung neuer Geschäftsmodelle (ausführlich: Degenhart/Nestle 2014). Hierzu gehören neben der Erweiterung des Angebots um Energiedienstleistungen - eine Produktstrategie, die im Übrigen auch Stadtwerke und andere Energieversorgungsunternehmen im Zuge der Energiewende diskutieren und teilweise bereits umsetzen (Berlo/Wagner 2011; BDEW/EY 2015) - auch die stärkere Verknüpfung von Produktion und Konsumtion (Kle- 
misch 2014a: 23). Mit der Vermarktung selbst erzeugten Stroms an die Mitglieder wird einer der wesentlichen Genossenschaftsgedanken wieder in den Vordergrund gerückt. Gleichzeitig wird damit die energiepolitisch diskutierte Verpflichtung zur Direktvermarktung aufgegriffen, wobei dies allerdings durch verschiedene Regelungen (unter anderem Zahlung der EEG-Umlage) erschwert wird.

Vor allem aus geographischer Perspektive stellt sich angesichts dieser Überlegungen die Frage, inwieweit eine solche Entwicklung noch dezentral stattfinden kann bzw. inwieweit sie mit einer regionalen Orientierung von Genossenschaften vereinbar ist. Die aktuelle Situation stellt für den relativ jungen Sektor der auf erneuerbare Energien fokussierten Energiegenossenschaften einen möglichen Wendepunkt dar. Die Innovationsforschung und konkret die innovationsbiographische Perspektive bieten eine geeignete theoretische Grundlage für eine empirische Analyse der bisherigen und möglicher zukünftiger Entwicklungen.

\subsection{Energiegenossenschaften als soziale Innovation und der Ansatz der Innovationsbiographie}

Die auf erneuerbare Energien fokussierten Energiegenossenschaften stellen - im Gegensatz zu den technischen Innovationen der Stromgewinnung aus erneuerbaren Energien - eine soziale Innovation dar. Soziale Innovationen sind neue bzw. neu konfigurierte soziale Praktiken, die von bisherigen Praktiken abweichen und für bestimmte Probleme bzw. Herausforderungen, hier die Umsetzung und Finanzierung der Energiewende, eine (bessere) Lösung darstellen sollen (Howaldt/Schwarz 2010: 89). Soziale Innovationen werden als geeignet bzw. notwendig angesehen, neuen gesellschaftlichen Herausforderungen zu begegnen und einen „gesellschaftlichen Anschluss an technische Innovationen zu gewährleisten“ (Blättel-Mink 2008: 286); insbesondere für die Transformation in Richtung auf mehr Nachhaltigkeit wird ihnen eine große Bedeutung beigemessen (Howaldt/ Schwarz 2010: 91; Schwarz/Birke/Beerheide 2010: 166). Während Genossenschaften, auch im Energiesektor, keine grundlegende Neuerung darstellen, sind die hier betrachteten Energiegenossenschaften vor allem deshalb innovativ, weil sie Bürgern die direkte Beteiligung an ErneuerbareEnergien-Anlagen ermöglichen, welche ins Stromnetz einspeisen. Bürger nutzen also eine existierende soziale Institution, die Genossenschaftsform, im Kontext der Energiewende und des liberalisierten Energiemarkts, um einer neuen gesellschaftlichen Herausforderung zu begegnen (Howaldt/ Schwarz 2010: 91; Elsen 2012). Energiegenossenschaften treten damit in Konkurrenz zu Energieversorgungsunternehmen und anderen gewerblichen Erzeugern und haben das Akteursspektrum in der Energiewirtschaft grundlegend verändert (Trend:research/KNI 2011).
Zur Analyse der Entstehung und Veränderung der sozialen Innovation Energiegenossenschaft wird im Folgenden der Ansatz der Innovationsbiographie verwendet, der der Kontextabhängigkeit sowie den Eigengesetzlichkeiten und Spezifika sozialer Innovationen besonders gut Rechnung tragen kann (Howaldt/Schwarz 2010: 93, 97). Der Ansatz wurde von Werner Rammert (2000) erarbeitet und auf Innovationsprozesse im Bereich erneuerbarer Energien bereits erfolgreich angewandt (Bruns/Köppel/Ohlhorst et al. 2008; Bruns/Ohlhorst/Wenzel et al. 2011). Er stellt die jeweils individuelle und interpretierte Geschichte einer Innovation - hier: der Energiegenossenschaften - mit ihren Brüchen, Wendepunkten, Momenten des Erfolgs und des Scheiterns im Innovationsprozess in den Mittelpunkt der Analyse. Er löst damit die lange dominierende Auffassung ab, Innovationsprozesse würden universellen Mustern folgen, die sich entlang einer bestimmten Phasenabfolge vollziehen (Thomi/Werner 2001: 203). Vielmehr beeinflussen je spezifische sozioökonomische und technische Faktoren den Innovationsverlauf, wodurch dieser bestimmte Charakteristika aufweist. Bruns und andere unterscheiden zwischen verschiedenen Phasentypen wie Pionier-, Aufbruch- und Boomphasen sowie Phasen der Konsolidierung und Stagnation nach einem Entwicklungsknick, die den Fortgang des Innovationsverlaufs jeweils spezifisch beschreiben (Bruns/Köppel/ Ohlhorst et al. 2008: 26 ff.; Bruns/Ohlhorst/Wenzel et al. 2011: 381 ff.).

Mit seiner Fokussierung auf innovationsbiographische Brüche und Wendepunkte ist dieser Ansatz geeignet, die (zunächst) dynamische Entwicklung von Energiegenossenschaften rekonstruierend-analytisch zu erklären sowie außerdem prospektiv-gestalterische Aspekte für die zukünftige Entwicklung herauszuarbeiten (Rammert 2000: 41; Bruns/Köppel/Ohlhorst et al. 2008: 24 f.; Bruns/Ohlhorst/ Wenzel et al. 2011: 11 f.; Ohlhorst/Schön 2015: 260). Von besonderem Interesse im Kontext der Energiegenossenschaften ist aktuell die Phase der Konsolidierung und Stagnation nach einem Entwicklungsknick. Dieser wird häufig ausgelöst durch einen ordnenden Eingriff aus dem Umfeld - etwa durch neue Regulierungen, veränderte Kreditvergabepraktiken, Rechtsunsicherheiten durch anhängige Klagen etc. - als eine Reaktion auf Verwerfungen in Folge einer „zu erfolgreichen“ Boomphase und kann eine umfassende Reorganisation des gesamten Sektors einleiten.

Die Betrachtung des Energiegenossenschaftssektors aus innovationsbiographischer Perspektive soll in diesem Beitrag Aufschluss über folgende Fragen geben: In welcher Innovationsphase befinden sich die Energiegenossenschaften nach der jüngsten Reform des EEG? Welche Entwicklungsmöglichkeiten zeichnen sich für Energiegenossenschaften ab? Schließlich soll die Diskussion dieser Aspekte helfen, die zentrale Frage dieses Beitrags zu beantworten: Wie können die Energiegenossenschaften angesichts des sich ver- 
ändernden Marktdesigns weiterhin erfolgreich wirtschaften und dabei ihre positiv konnotierte regionale Orientierung erhalten? Bevor mögliche zukünftige Entwicklungen genauer betrachtet und beschrieben werden können, ist es notwendig, den bisherigen Verlauf und die Strukturen im deutschen Energiegenossenschaftssektor besser zu verstehen.

\section{Entwicklung und Strukturen von Energiegenossenschaften in Deutschland aus innovationsbiographischer Perspektive}

Nach einem historischen Rückblick und Überblick über die jüngsten Entwicklungen von Energiegenossenschaften (3.1) sowie Analysen zu den sich verändernden Größenstrukturen anhand von Mitgliederzahlen und Investitionsvolumina (3.2) und der Bedeutung einer regionalen Orientierung (3.3) werden diese Informationen innovationsbiographisch interpretiert und für die Herleitung eines Phasenmodells genutzt (3.4). Insgesamt wird deutlich, dass Energiegenossenschaften sowohl hinsichtlich ihrer Größe als auch ihrer Reichweite dynamisch gewachsen und bereits heute sehr vielfältig sind. Sie weisen damit unterschiedliche Ausgangsvoraussetzungen für die Bewältigung der anstehenden Herausforderungen auf.

\subsection{Historischer Rückblick und jüngere Entwicklungen}

Historisch gesehen bilden Genossenschaften in der Energiewirtschaft keine Neuerung, die erst im Zusammenhang mit erneuerbaren Energien aufgetreten ist. Ihre Geschichte geht auf die Mitte des 19. Jahrhunderts zurück und schon im frühen 20. Jahrhundert waren sogenannte Elektrizitätsgenossenschaften in ländlichen Regionen Deutschlands als Selbsthilfeorganisationen weit verbreitet (Volz 2012a; Holstenkamp/Degenhart 2013). Sie standen oft am Beginn der lokalen Energieversorgung, wurden aber zwischenzeitlich meist durch regionale oder sogar überregionale Energieversorgungsunternehmen abgelöst bzw. übernommen. Mit dem Aufschwung der erneuerbaren Energien in den letzten Jahren und Jahrzehnten haben Energiegenossenschaften wieder an Bedeutung gewonnen, und dies nicht allein in Deutschland mit seinen bislang günstigen Förderbedingungen, sondern auch in Dänemark, Kanada, Großbritannien, der Schweiz und den USA (Viardot 2013; siehe auch Walker 2008; Schreuer/Weismeier-Sammer 2010).

In Deutschland ist die Zahl der Energiegenossenschaften vor allem seit 2005 rasant angestiegen (Maron/Maron 2012; DGRV 2013; Holstenkamp/Müller 2013), von weniger als $100 \mathrm{im}$ Jahr 2005 auf fast 1000 Ende 2014 (973 gemäß Müller/Holstenkamp 2015). Allein die seit 2006 gegründeten Energiegenossenschaften hatten im Jahr 2014 rund 145.000 Mitglieder, davon waren 130.000 Privatpersonen
(90\%); sie verfügten über rund 470 Mio. Euro Eigenkapital und ihre Investitionen in erneuerbare Energien beliefen sich auf 1,35 Mrd. Euro (DGRV 2014). Darin enthalten ist Fremdkapital, vor allem in Form von Krediten von Genossenschaftsbanken (64\%); daneben spielten, zumindest im Vorjahr, auch Förderkredite eine bedeutende Rolle (DGRV 2013).

\subsection{Größenstrukturen im Wandel}

Die Größe einer Energiegenossenschaft und ihr Wachstum können anhand verschiedener Kriterien untersucht werden. Die besten Daten liegen zu den Mitgliederzahlen und den Investitionssummen vor. Hier sind vor allem die auf der Basis von Befragungen erhobenen Daten von Volz (2012a; $n=119$ aus einer Grundgesamtheit von 263 bezogen auf das Jahr 2010), dem DGRV (2013; $n=213$ aus einer Grundgesamtheit von $656 \mathrm{im}$ Jahr 2013) sowie einer eigenen Erhebung ( $n=181$ aus einer Grundgesamtheit von $586 \mathrm{im} \mathrm{Jahr}$ 2014; vgl. Schmole 2014) interessant und zeigen deutliche Veränderungen im Zeitverlauf.

So ist die Zahl der Mitglieder zum Zeitpunkt der Gründung meist relativ klein, aber sie war 2013 bereits deutlich höher als im Jahr 2010: Bei Volz (2012a) hatten noch rund zwei Drittel der befragten Energiegenossenschaften nicht mehr als 20 und nur 4 Prozent über 100 Gründungsmitglieder; bei der Befragung des DGRV (2013) lagen die entsprechenden Zahlen bereits bei 49 bzw. 9 Prozent. Bedeutsamer als die steigende Zahl der Gründungsmitglieder ist jedoch das durchschnittlich starke Mitgliederwachstum nach der Gründung. Dies verdeutlichen die Mitgliederzahlen der befragten Energiegenossenschaften zum Zeitpunkt der jeweiligen Erhebungen, die erkennbar über jenen zum Gründungszeitpunkt liegen; auch zwischen den Erhebungszeitpunkten kommt es zu einer Anteilsverschiebung in Richtung der größeren Genossenschaften (siehe Tab. 1). So liegt zwar der Anteil der befragten Energiegenossenschaften mit bis zu 50 Mitgliedern sowohl 2010 als auch 2014 um 18 Prozent, was vermutlich auf die hohe Zahl an Neugründungen zurückzuführen ist. Im gleichen Zeitraum ist der Anteil der Energiegenossenschaften mit mehr als 200 Mitgliedern jedoch von einem Zehntel auf ein Drittel gestiegen; die absolute Zahl der Mitglieder hat sich damit angesichts der steigenden Zahl der Energiegenossenschaften insgesamt mehr als verfünffacht.

Auch die Investitionsvolumina (Eigen- und Fremdkapital) der Energiegenossenschaften sind zwischen 2010 und 2013 größer geworden, allerdings sind die Veränderungen hier weniger deutlich als bei den Mitgliederzahlen. So hat sich der Anteil der befragten Energiegenossenschaften mit einem niedrigen Investitionsvolumen von maximal 1 Mio. Euro von 65 auf 61 Prozent verringert, gleichzeitig ist der Anteil der Energiegenossenschaften mit hohen Investitions- 
Tab. 1 Verteilung der Energiegenossenschaften auf Größenklassen nach Mitgliederzahlen zum jeweiligen Befragungszeitpunkt 2010,2013 und 2014

\begin{tabular}{llllll}
\hline Bezugsjahr & 2010 & 2013 & 2014 & \\
\hline & $(n=119)$ & $(n=213)$ & $\begin{array}{l}\text { Insgesamt } \\
(n=181)\end{array}$ & $\begin{array}{l}\text { Davon: Regionale } \\
\text { Energiegenossenschaften } \\
(n=118)\end{array}$ & $\begin{array}{l}\text { Davon: Überregionale } \\
\text { Energiegenossenschaften } \\
(n=63)\end{array}$ \\
Bis 20 & $7,5 \%$ & $13 \%$ & $8,3 \%$ & $8,5 \%$ & $7,9 \%$ \\
$21-50$ & $10,9 \%$ & & $9,9 \%$ & $9,3 \%$ & $11,1 \%$ \\
$51-100$ & $43,7 \%$ & $24 \%$ & $20,4 \%$ & $22,0 \%$ & $17,5 \%$ \\
$101-200$ & $27,7 \%$ & $37 \%$ & $28,2 \%$ & $33,1 \%$ & $19,0 \%$ \\
$201-300$ & $10,1 \%$ & $14 \%$ & $16,0 \%$ & $14,4 \%$ & $19,0 \%$ \\
$301-400$ & & $6 \%$ & $7,2 \%$ & $6,8 \%$ & $7,9 \%$ \\
Über 400 & & $6 \%$ & $9,9 \%$ & $5,9 \%$ & $17,5 \%$ \\
Summen $^{\mathrm{b}}$ & $100 \%$ & $100 \%$ & $100 \%$ & $100 \%$ & $100 \%$ \\
\hline
\end{tabular}

${ }^{\mathrm{a}}$ Gemäß unserer Definition (vgl. Abschnitt 3.3) verfügen regionale Energiegenossenschaften nur in ihrer eigenen Gemeinde bzw. im eigenen Landkreis über Anlagen, während überregionale Energiegenossenschaften auch darüber hinaus (inklusive Ausland) Anlagen besitzen

${ }^{\mathrm{b}}$ Aufgrund von Rundungsdifferenzen ergeben die aufsummierten Prozentanteile nicht immer genau 100 Prozent.

Quellen: Volz 2012a: 517, Abb. 2; DGRV 2013; eigene Befragung

volumina gestiegen. Im Jahr 2010 hatten erst 9 Prozent der befragten Energiegenossenschaften ein Investitionsvolumen über 4 Mio. Euro. Im Jahr 2013 waren es 10 Prozent mit Investitionsvolumina von mindestens 5 Mio. Euro, davon sogar ein Fünftel $(2 \%)$ mit mehr als 10 Mio. Euro. ${ }^{3}$

Diese Zahlen und vor allem die Anteile großer Genossenschaften sind vor dem Hintergrund einer insgesamt deutlich steigenden Zahl von Energiegenossenschaften, also einer regen Neugründungsaktivität mit vermutlich eher geringen Anfangsgrößen, zu interpretieren. Konkret bedeutet dies, dass existierende Genossenschaften stark gewachsen sind und die absolute Zahl der Energiegenossenschaften mit großen Mitgliederzahlen bzw. hohen Investitionsvolumina deutlich zugenommen hat. Der gleichzeitig weiterhin hohe Anteil sowie die absolut ebenfalls steigende Zahl von Energiegenossenschaften mit geringen Investitionsvolumina und Mitgliederzahlen deuten auf eine wachsende Disparität zwischen kleinen und großen Energiegenossenschaften hin, während die Zahl der Energiegenossenschaften mittlerer Größenordnung relativ dazu zurückgeht.

\subsection{Regionale Orientierung verliert an Bedeutung}

Die existierende Literatur hebt die besondere Rolle regional orientierter Energiegenossenschaften für eine auf dezentrale Strukturen der Erzeugung und des Verbrauchs von Energie orientierte Energiewende hervor (Jakubowski/Koch

\footnotetext{
3 Ähnliche Ergebnisse aus einer anderen Quelle werden in Yildiz/ Rommel/Debor et al. (2015: 63, Abb. 2) zitiert. Demnach verringerte sich der Anteil jener Genossenschaften mit einem Finanzkapital von bis zu einer Million Euro von 69 Prozent im Jahr 2010 auf nur noch 65 Prozent im Jahr 2012. Hingegen ist der Anteil der Energiegenossenschaften mit einem Finanzkapital von mehr als 2 Millionen Euro von 14 Prozent im Jahr 2010 auf 20 Prozent im Jahr 2012 gestiegen.
}

2012: 475). Die regionale Orientierung und ihre demokratische Verfasstheit mit überschaubaren Mitbestimmungsstrukturen werden als wesentliche Merkmale und Erfolgsfaktoren dieser Rechts- und Organisationsform betont (vgl. Abschnitt 2.2). Tatsächlich ist aber nicht nur die Größenstruktur von Energiegenossenschaften im Wandel und von Vielfalt geprägt, sondern auch hinsichtlich der regionalen Orientierung gibt es ein breites Spektrum verschiedener Ausprägungen. Obwohl die Datenbasis zur regionalen Orientierung von Energiegenossenschaften weniger umfangreich ist, zeigen die verfügbaren Daten doch, dass die Zahl überregional orientierter Energiegenossenschaften zumindest in absoluten Zahlen zunimmt.

Im Jahr 2010 war gemäß Volz (2012a: 520) die „Mitgliedschaft [...] in rund $56 \%$ der befragten Energiegenossenschaften regional beschränkt". Eine allerdings nur 30 Energiegenossenschaften umfassende Telefonbefragung von Trend.research/Leuphana Universität Lüneburg (2013: 61 f., Abb. 24) kommt zu einem ähnlichen Ergebnis: Demzufolge verfolgen fast 90 Prozent der befragten Energiegenossenschaften das Ziel, ausschließlich (30\%) oder vornehmlich (57\%) Mitglieder aus der Region aufzunehmen, und „nur zehn Prozent gaben an, überregional tätig zu sein und deutschlandweit Mitglieder aufzunehmen“" (Trend.research/Leuphana Universität Lüneburg 2013: 61).

Auch im Jahr 2014 ist gemäß der eigenen Befragung die Mitgliedschaft bei den meisten Genossenschaften auf die regionale oder sogar lokale Ebene (Landkreis oder Gemeinde) begrenzt ( 94 Genossenschaften oder ca. $52 \%$; siehe Tab. 2). Von den verbleibenden ca. 48 Prozent haben immerhin 56 Genossenschaften (fast 31 Prozent der Grundgesamtheit) keinerlei räumliche Begrenzung der Mitgliedschaft. Eine noch stärkere regionale Beschränkung weisen die befragten Energiegenossenschaften hinsichtlich der An- 
Tab. 2 Räumliche Reichweite der Mitgliedschaft und der Anlagenstandorte deutscher Energiegenossenschaften (Bezugsjahr: 2014; $n=181$ )

\begin{tabular}{|c|c|c|c|c|c|c|c|}
\hline & & \multicolumn{6}{|c|}{ Reichweite Anlagenstandorte } \\
\hline \multirow{8}{*}{$\begin{array}{l}\text { Reichweite } \\
\text { Mitglie- } \\
\text { der }\end{array}$} & & Gemeinde & Landkreis & Bundesland & Deutschland & Keine Begrenzung & Gesamt \\
\hline & Gemeinde & 39 & 2 & 1 & 1 & 5 & 48 \\
\hline & Landkreis & 8 & 30 & 2 & 3 & 3 & 46 \\
\hline & Bundesland & 1 & 3 & 4 & 1 & 1 & 10 \\
\hline & Deutschland & 4 & 2 & 2 & 11 & 2 & 21 \\
\hline & Keine Begrenzung & 8 & 21 & 2 & 4 & 21 & 56 \\
\hline & Gesamt & 60 & 58 & 11 & 20 & 32 & 181 \\
\hline & $\begin{array}{l}\text { Definition (vgl. } \\
\text { Abschnitt 4.1) }\end{array}$ & \multicolumn{2}{|c|}{$\begin{array}{l}\text { Regionale Energiegenossenschaften } \\
(118 ; 65 \%)\end{array}$} & \multicolumn{4}{|c|}{ Überregionale Energiegenossenschaften (63; $35 \%)$} \\
\hline
\end{tabular}

Quelle: Eigene Befragung

lagenstandorte auf: 65 Prozent verfügen nur in der eigenen Region über Anlagen. Von den verbleibenden 35 Prozent weist immerhin knapp die Hälfte (32 Genossenschaften oder $17 \%$ der Grundgesamtheit) keinerlei räumliche Begrenzung auf. Trotz der nach wie vor großen Bedeutung regionaler Strukturen und Bindungen ist im Jahr 2014 bereits rund die Hälfte der befragten Energiegenossenschaften nicht mehr (nur) regional orientiert. Der Anteil der Genossenschaften mit deutschlandweiter oder sogar internationaler Ausrichtung bezüglich Mitgliedschaft und Anlagenstandorten liegt bereits bei einem knappen Fünftel (38 von 181; siehe Tab. 2).

\subsection{Innovationsbiographische Interpretation und Phasenmodell}

Der innovationsbiographische Verlauf der Institution Energiegenossenschaft wird durch diese quantitativen Veränderungen gut verdeutlicht: Sowohl die Zahl der Gründungen als auch die absolute Zahl der Mitglieder sind vor allem seit 2005 stark angestiegen und die Investitionsvolumina sind größer geworden. Die Zahl der Energiegenossenschaften mit großen Mitgliederzahlen (über 200) bzw. hohen Investitionsvolumina hat spätestens ab 2010 deutlich zugenommen, wobei sich eine wachsende Disparität zwischen kleinen (bis 50 Mitglieder) und großen Energiegenossenschaften abzeichnet, während die Zahl der Energiegenossenschaften mittlerer Größenordnung (51 bis 200 Mitglieder) relativ dazu zurückgeht. Rund die Hälfte der Energiegenossenschaften ist nicht (mehr) nur regional ausgerichtet. Nimmt man die Anzahl der Neugründungen bzw. Neueintragungen von Energiegenossenschaften als Indikatoren, so lassen sich bis 2014 grob vier Innovationsphasen unterscheiden (siehe Abb. 1):

- Bis 2007 befand sich der Sektor der Energiegenossenschaften in der Pionierphase, in der es nur vereinzelt zu Gründungen kam. Als Hintergrund für die ,Wiederentdeckung“ des genossenschaftlichen Modells im Energie- sektor ist neben den günstigen Förderbedingungen durch das EEG die Novellierung des Genossenschaftsgesetzes im Jahr 2006 anzuführen, die insbesondere den Aufwand für Gründung und Geschäftsbetrieb verringerte.

- Das Jahr 2008 kann mit rund 50 Gründungen als Aufbruchphase eingeordnet werden. Die Idee der Energiegenossenschaft findet Verbreitung, Nachahmer werden aktiv. Relativ hohe Einspeisevergütungen bei sinkenden Anlagenpreisen machen die Investitionen in Erneuerbare-Energien-Anlagen nicht nur für private Anlagenbetreiber, sondern zunehmend auch für Energiegenossenschaften attraktiv.

- 2009 markiert mit etwas mehr als doppelt so vielen Neugründungen als 2008 dann schon den recht schnellen Übergang in eine Boomphase. Jetzt haben sich die Vorteile der sozialen Innovation Energiegenossenschaft weit herumgesprochen, sodass viele daran partizipieren wollen. Mit rund 180 Neugründungen jährlich findet der Boom im Jahr 2011 seinen Höhepunkt. In den Jahren 2012 und 2013 geht die Neugründungsaktivität leicht zurück, sodass man von einer auslaufenden Boomphase sprechen kann. Gleichzeitig wächst ein Teil der existierenden Genossenschaften hinsichtlich Mitgliederzahl und räumlicher Reichweite. Neben den im Rahmen der EEG-Novellen von 2009 und 2012 abgesenkten Einspeisevergütungen (bei allerdings weiter stark sinkenden Preisen v. a. für Fotovoltaik-Anlagen) erklären auch die wachsenden Probleme, geeignete Flächen zu finden, die Stagnation. Ab 2012 beeinträchtigt außerdem die antizipierte und 2014 vollzogene grundlegende Neuregelung des EEG die Neugründungstätigkeit.

- Im Jahr 2014 bricht die Zahl der Neugründungen ein und geht von noch über $100 \mathrm{im}$ Jahr 2013 um mehr als 50 Prozent im Jahr 2014 zurück und ist im Jahr 2015 noch weiter zurückgegangen, womit sich der Sektor klar in einer Phase der Konsolidierung und Stagnation befindet (Netzwerk Energiewende jetzt e. V. 2016). 
Es deutet alles darauf hin, dass sich der Energiegenossenschaftssektor mit einem Entwicklungsknick in seiner Innovationsbiographie auseinandersetzen muss, der durch die ab 2012 antizipierten Neuregelungen des EEG 2014 ausgelöst wurde (vgl. dazu die Abschnitte 2.3 bis 2.5). Damit ist der Energiegenossenschaftssektor in eine kritische Innovationsphase eingetreten. In Bezug auf die soziale Innovation Energiegenossenschaft bedeutet dies, dass diese sich anpassen und wandeln muss, um weiter eine vergleichbar bedeutsame Rolle im Energiesektor zu spielen. Vor diesem Hintergrund ist es interessant, sich näher mit qualitativen Aspekten und Veränderungen $\mathrm{zu}$ beschäftigen und dabei zwischen regionalen und überregionalen Energiegenossenschaften zu unterscheiden.

\section{Regionale und überregionale Energiegenossenschaften: Unterschiede, Fallbeispiele und Entwicklungsmöglichkeiten}

Die bisherigen Analysen verdeutlichen nicht nur, dass das Spektrum deutscher Energiegenossenschaften vielfältig ist, sondern auch, dass finanzielle Anreize und Rahmenbedingungen für ihre Entstehung und Entwicklung maßgeblich sind. Die sich verändernden Rahmenbedingungen (vgl. Abschnitt 2.3) werden die Struktur und die Tätigkeit von Energiegenossenschaften - und damit den genossenschaftlichen Beitrag zur Finanzierung der Energiewende - spürbar beeinflussen. Insbesondere dürften, so die Hypothese (siehe Einleitung), die neuen Rahmenbedingungen eine Schlechterstellung von regional orientierten bzw. kleinen gegenüber größeren, überregional agierenden Genossenschaften zur Folge haben. Diese Vermutung einer unterschiedlichen Struktur und Entwicklung regionaler und überregionaler Energiegenossenschaften wird im Folgenden auf der Basis von Befragungsergebnissen (4.1) sowie anhand von Fallbeispielen (4.2) näher untersucht. Bezugszeitpunkt ist das Jahr 2014, welches das Ende der Boomphase und den Beginn einer durch Konsolidierung und Stagnation geprägten kritischen Innovationsphase nach einem Entwicklungsknick in der Innovationsbiographie der sozialen Innovation Energiegenossenschaft markiert (vgl. Abschnitt 3.4).

\subsection{Differenzierung und Unterschiede zwischen regionalen und überregionalen Energiegenossenschaften in Deutschland}

Die im Folgenden getroffene Unterscheidung zwischen regionalen und überregionalen Energiegenossenschaften sowie die entsprechenden Analysen selbst erhobener Befragungsdaten basieren auf der Reichweite der Anlagenstandorte. Diese kann als Ausdruck des Geschäftsmodells einer
Genossenschaft interpretiert werden, während die räumliche Herkunft der Mitglieder durch die Leitungsgremien nur bedingt beeinflusst werden kann, da diese trotz regionalem Fokus z. B. nach einem Umzug Mitglied bleiben (können). Als regionale Energiegenossenschaften bezeichnen wir im Folgenden solche, die (zum Befragungszeitpunkt) ausschließlich Anlagen im eigenen Landkreis (bzw. sogar nur in der eigenen Gemeinde) haben, als überregionale dagegen solche mit Anlagen bzw. Aktivität (auch) außerhalb des eigenen Landkreises (siehe Tab. 2).

Zunächst ist festzuhalten, dass überregionale Genossenschaften größer sind als regionale Genossenschaften. Sie haben höhere Mitgliedszahlen (siehe die letzten beiden Spalten von Tab. 1) und weisen bei den Investitionssummen höhere Durchschnittswerte auf (3,7 Mio. Euro versus 2,1 Mio. Euro). Mit Ausnahme des Wärmebereichs (Erzeugung, Wärmenetze), der eine Domäne regionaler Energiegenossenschaften ist, sind überregionale Energiegenossenschaften außerdem hinsichtlich ihrer Tätigkeiten breiter aufgestellt (siehe Abb. 2). Dies gilt für ihre Stromerzeugung und -vermarktung, die weniger stark auf die Fotovoltaik bzw. die Vergütung nach EEG fokussiert ist als bei den regionalen Energiegenossenschaften; auch bieten sie öfter Leistungen jenseits der Erzeugung und Vermarktung von Strom oder Wärme an (z. B. Energieeffizienz- und Einsparcontracting-Dienstleistungen). Bei der Stromvermarktung ist interessant, dass hier vor allem die Vor-Ort-Vermarktung an Dritte in räumlicher Nähe ohne Leitung über öffentliche Netze am häufigsten genannt wurde, und zwar von fast einem Drittel aller überregionalen Energiegenossenschaften, aber immerhin auch von gut einem Fünftel der regionalen Energiegenossenschaften. Damit sind offensichtlich überregionale eher als regionale Energiegenossenschaften dazu in der Lage bzw. bereit, Stromproduktion und -konsumtion miteinander zu verknüpfen und damit einen zentralen Genossenschaftsgedanken zu realisieren (vgl. Abschnitt 2.1).

Überregionale Energiegenossenschaften kooperieren auBerdem öfter mit anderen Energiegenossenschaften als ihre regionalen Pendants, aber auch mit weiteren Akteuren (Abb. 3). Insgesamt weisen über 60 Prozent der überregionalen, aber nur unter 40 Prozent der regionalen Energiegenossenschaften Kooperationen mit weiteren Akteuren auf. Interessant ist, dass für regionale Energiegenossenschaften Landwirte die wichtigste Einzelgruppe unter den möglichen Kooperationspartnern darstellen; der Grund hierfür liegt vermutlich darin, dass ein relativ hoher Anteil der regionalen Energiegenossenschaften (auch) Wärmegenossenschaften sind. Auffällig ist außerdem der Unterschied bei Kooperationen mit Stadtwerken: Die weitaus überwiegende Zahl der befragten regionalen Energiegenossenschaften nennt sie nicht als Kooperationspartner, während dies immerhin ein Drittel der überregionalen Energiegenossenschaften tun. Angesichts der Überlegungen zu den Vorteilen 
Abb. 2 Tätigkeitsfelder regionaler und überregionaler Energiegenossenschaften (EG) in Deutschland im Vergleich, $2014(n=181)$ (Quelle: Eigene Befragung)

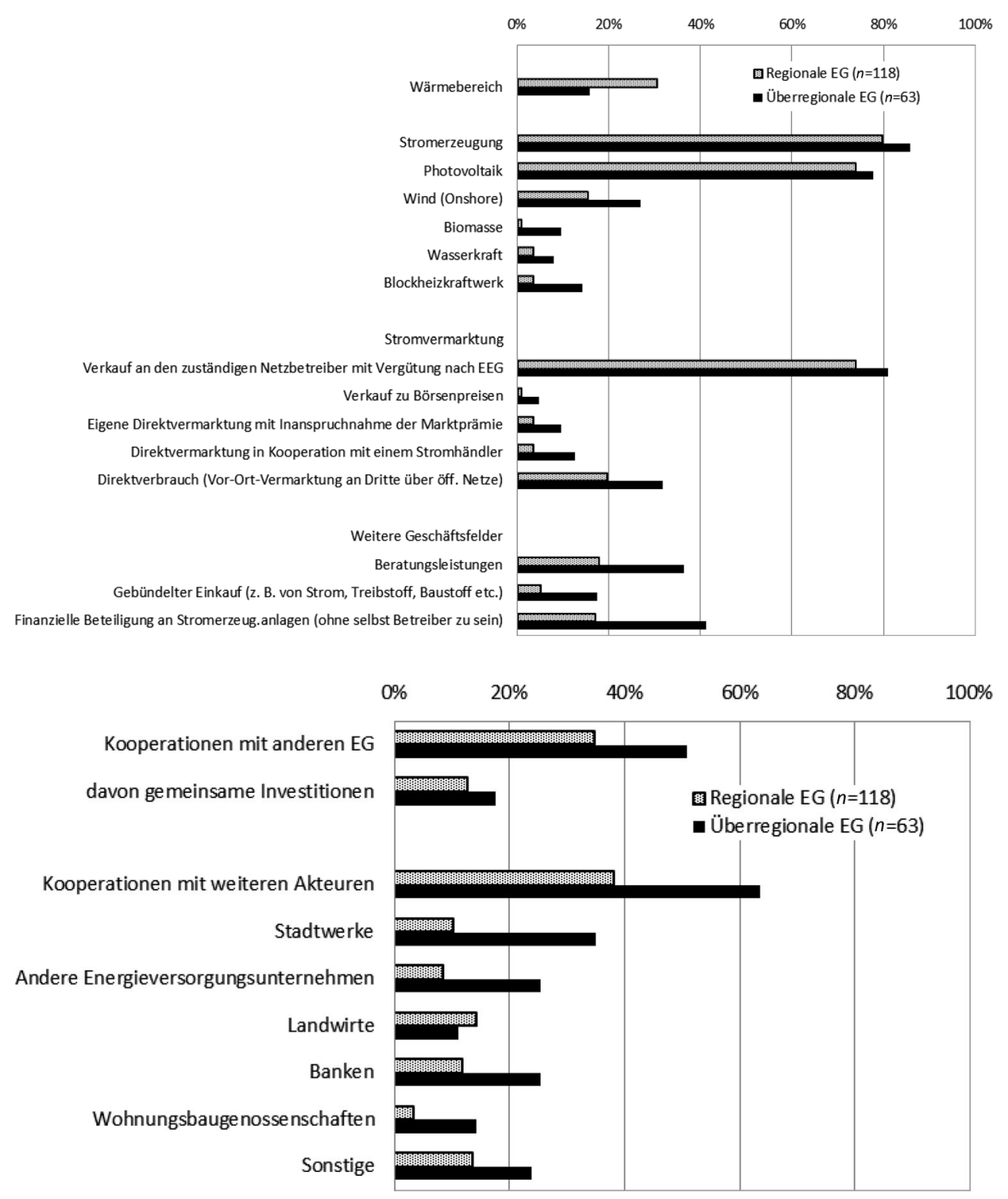

Abb. 3 Kooperationen regionaler und überregionaler Energiegenossenschaften (EG) in Deutschland im Vergleich, 2014 $(n=181$, Quelle: Eigene Befragung) einer regionalen Orientierung in Abschnitt 2.2 ist dies ein interessanter Befund, der verdeutlicht, dass überregional aktive Energiegenossenschaften durchaus auch Kooperationen auf regionaler Ebene eingehen bzw. erfolgreich eingehen können.

Die Analysen verdeutlichen, dass überregionale Energiegenossenschaften nicht nur bezogen auf die Möglichkeiten, Größenvorteile zu realisieren, sondern auch im Hinblick auf die Diversifizierung von Risiken besser aufgestellt sind als regionale Energiegenossenschaften. Sie sind damit - im Sinne der Überlegungen in Abschnitt 2.3 - stärker professionalisiert und damit potenziell eher in der Lage, die mit dem EEG 2.0 verbundenen Herausforderungen zu bewältigen. Dieser Punkt wird im Folgenden anhand eines Vergleichs einer regionalen und einer überregionalen deutschen Energiegenossenschaft aufgegriffen und relativiert, die bei- de mit jeweils spezifischen Geschäftsmodellen erfolgreich sind.

\subsection{Energiegenossenschaften mit Diversifizierung bzw. Direktvermarktung weiterhin erfolgreich?}

Trotz des sich abzeichnenden deutlichen Rückgangs der Förderung sowie auch der zurückgehenden Neugründungszahlen (siehe Abb. 1) wollen einzelne Genossenschaften mit spezifischen Geschäftsmodellen weiter wachsen bzw. auch zukünftig ihre Geschäftstätigkeit weiter ausbauen. Als Beispiele hierfür werden nachfolgend zwei unterschiedlich strukturierte Genossenschaften vorgestellt, die aufgrund ihres Erfolgs besonders bekannt sind (Flieger 2010; Flieger 2011; Klemisch 2014b). Die Entwicklungsdynamiken dieser beiden - keineswegs repräsentativen - Energiege- 
Tab. 3 Zwei Fallbeispiele deutscher Energiegenossenschaften

\begin{tabular}{|c|c|c|}
\hline & Greenpeace Energy eG ${ }^{a}$ & Energiegenossenschaft Odenwald eG $\mathrm{a}^{\mathrm{a}}$ \\
\hline Gründungsjahr: & 1999 & 2009 \\
\hline $\begin{array}{l}\text { Mitgliederzahl: } \\
\text { - kurz nach der Gründung } \\
\text { - } 2013\end{array}$ & $\begin{array}{l}5.732(2000) \\
22.665\end{array}$ & $\begin{array}{l}203 \text { (Ende 2009) } \\
2.515\end{array}$ \\
\hline \multicolumn{3}{|l|}{ Finanzielle Kennziffern (2013): } \\
\hline - Genossenschaftsanteile & 12,5 Mio. $€$ & 9,4 Mio. $€$ \\
\hline - Bilanzsumme & 29,4 Mio. $€$ & 37,6 Mio. $€$ \\
\hline - Umsatz & 103,5 Mio. $€$ & 1,5 Mio. $€$ \\
\hline $\begin{array}{l}\text { Räumliche Orientierung in Bezug auf Mitglie- } \\
\text { der, Kunden und Anlagen }\end{array}$ & Für alle drei überregionale Orientierung & Für alle drei regionale Orientierung \\
\hline
\end{tabular}

${ }^{a}$ Ohne Tochtergesellschaften

Quellen: Greenpeace Energy 2014a: 2; Greenpeace Energy 2014b: 4; EGO 2014a: 4 ff.; EGO 2014b: 2, 6, 8

nossenschaften illustrieren die große Bedeutung der Diversifizierung bzw. der Direktvermarktung. Mit der Greenpeace Energy eG wurde hier eine überregionale Energiegenossenschaft gewählt, die 1999 als bundesweiter Ökostromanbieter gegründet wurde, also ihren Ursprung im Vertrieb hat (Greenpeace Energy 2014a). Hingegen hat die erst 2009 gegründete Energiegenossenschaft Odenwald (EGO) zunächst, wie viele andere regionale Energiegenossenschaften, vor allem auf die Stromerzeugung durch Fotovoltaik sowie die Vergütung gemäß EEG gesetzt, gehört inzwischen aber hinsichtlich Mitgliederzahl und auch Bilanzsumme zu den größeren Energiegenossenschaften (EGO 2014a; EGO 2014b).

Als Gemeinsamkeiten im Entwicklungs- und Innovationsprozess der beiden Fallbeispiele sind das Größenwachstum - so sind bei beiden Energiegenossenschaften die Bilanzsumme und Mitgliederzahl zwischen 2010 und 2013 kontinuierlich gestiegen - sowie die Ausweitung des jeweiligen Tätigkeitsspektrums hervorzuheben. Außerdem haben beide festangestellte Mitarbeiter und weisen damit professionalisierte Strukturen auf. Dennoch stehen sie hinsichtlich ihrer regionalen Orientierung an unterschiedlichen Enden des Spektrums deutscher Energiegenossenschaften (siehe hier und im Folgenden Tab. 3). Greenpeace Energy eG gehört zu den Marktführern im Bereich Ökostromvermarktung in Deutschland, befindet sich aber in einem Diversifizierungsprozess von einem reinen Stromhändler in Richtung eigenständige Stromerzeugung, wobei nicht nur kleinteilige dezentrale Konzepte zur Anwendung kommen. Über die 2001 gegründete Tochtergesellschaft „Planet Energy“ engagiert sich die Greenpeace Energy eG in Projektierung, Bau und Betrieb von Energieproduktionsanlagen und hat als Fernziel, den gesamten verkauften Strom selbst zu erzeugen. Mit dem Verkauf von Windgas, also eines mittels Windstrom hergestellten Brenngases, wird außerdem ein Beitrag zur Entwicklung von Speichertechnologien geleistet; hinzu kommt ein explizites politisches Engagement für die Energiewende sowie für Forschung und Entwicklung im Bereich der erneuerbaren Energien (Greenpeace Energy 2014a: 2, 4, 6, 7).

Im Gegensatz zu Greenpeace Energy eG ist die Energiegenossenschaft Odenwald (EGO) klar regional orientiert. Es bestehen Partnerschaften mit verschiedenen lokalen Akteuren, die teilweise auch selbst Mitglied der Genossenschaft sind bzw. sogar zu den Initiatoren und Gründungsmitgliedern gehören (Volksbank Odenwald, die Städte Erbach und Michelstadt; außerdem seit 2012 die Stadt Griesheim) (EGO 2014b). Der weitaus überwiegende Teil der Mitglieder besteht jedoch aus Privatpersonen aus dem Odenwaldkreis, hinzu kommen Städte und Gemeinden aus der Region. Die EGO will explizit einen Beitrag zur dezentralen Energiewende sowie zur Stärkung der Region und ihrer Wirtschaftskraft durch erneuerbare Energien leisten und baut die entsprechenden Geschäftsfelder stetig weiter aus. Dabei werden nur Projekte verfolgt, die sich auch bei Einbezug eines Risikopuffers wirtschaftlich tragen und gleichzeitig von den Bürgern gewollt sind (EGO o.J.). So ist die Genossenschaft inzwischen in die Energieproduktion aus Windkraft und den Wärmemarkt eingestiegen, bietet weitere Energie(spar-) sowie Projektierungs- und sogar Immobilien-Dienstleistungen für Akteure vor Ort an; außerdem vermarktet sie seit 2013 selbst erzeugten Strom und auch eingekauftes Gas direkt vor Ort (EGO 2014a: 10; EGO 2014b: 5, 8, 11, 12).

Im Vergleich weisen Greenpeace Energy eG und EGO zwar sehr unterschiedliche Innovationsbiographien auf, die durch jeweils kontextspezifische Weiterentwicklungen der jeweiligen Geschäftsmodelle geprägt sind. Bei beiden bildet jedoch die Diversifizierung das für den bisherigen Erfolg vermutlich entscheidende Moment. Während die EGO vor allem ihr Leistungsspektrum mit Bezug auf die Region ausweitet und dabei auch in Bereiche jenseits des Energiesektors expandiert, erschließt sich die Greenpeace Energy eG weitere Schritte der Energie-Wertschöpfungskette und setzt dabei auf Größenvorteile und die bereits im Vertrieb erreichte Marktposition im nationalen Kontext. Bei beiden 
spielt die Vermarktung von Strom an die Mitglieder bzw. die Direktvermarktung von Energie eine wichtige Rolle. Beide richten sich vor allem an von ihrem jeweiligen Konzept überzeugte Mitglieder bzw. Kunden. Dabei steht bei Greenpeace Energy eG eher die politische Förderung der Energiewende und des Klimaschutzes im Vordergrund, während die EGO stärker auf die regionale Verbundenheit und Wertschöpfung in Zusammenhang mit einer dezentralen Energiewende setzt.

\section{Diskussion und Ausblick}

Der Sektor der Energiegenossenschaften hat sich in Deutschland vor dem Hintergrund garantierter Einspeisevergütungen etwa seit dem Jahr 2005 sehr dynamisch entwickelt und innerhalb weniger Jahre eine Aufbruch-, eine Wachstumsund eine Boomphase durchlaufen. Die im Jahr 2014 veränderten institutionellen und rechtlichen Bedingungen (EEGNovellierung) haben inzwischen zum Übergang in eine kritische Innovationsphase geführt. Sie werden als Hemmnis für die Weiterentwicklung des überwiegend kleinteilig und regional strukturierten Energiegenossenschaftssektors angesehen. Allerdings sind Energiegenossenschaften hinsichtlich ihrer Größe, regionalen Orientierung und ihrer Geschäftsfelder vielfältiger, als es die Debatte um die Probleme von Energiegenossenschaften mit den Veränderungen im institutionellen Kontext vermuten lässt. Welche Rolle Energiegenossenschaften im neuen Energiemarktdesign spielen werden, ist auch vor diesem Hintergrund schwer zu beantworten.

Die zukünftige energiewirtschaftliche und -politische Bedeutung von Energiegenossenschaften wird unter anderem davon abhängen, wie sich die einzelnen Energiegenossenschaften unter den neuen Rahmenbedingungen behaupten und positionieren. Und obwohl überregionale Energiegenossenschaften angesichts der rechtlichen $\mathrm{Si}$ tuation klare Vorteile besitzen, zeigen unsere Analysen, dass es auch für regionale Energiegenossenschaften erfolgversprechende Entwicklungsmöglichkeiten gibt. Damit ist die eingangs aufgestellte Hypothese zur notwendigen Veränderung von Strukturen und Reichweite der sozialen Innovation Energiegenossenschaft als Bedingung für einen auch in Zukunft signifikanten Beitrag zu einer dezentralen Energiewende zu relativieren. Insbesondere liegen in einer regionalen Orientierung von (einzelnen) Energiegenossenschaften auch Chancen für die erfolgreiche Fortsetzung der genossenschaftlichen Finanzierung der Energiewende, und sie kann unter bestimmten Bedingungen den Kern für ein erfolgreiches Geschäftsmodell bilden.

Mithilfe der innovationsbiographischen Perspektive konnte herausgearbeitet werden, dass eine erfolgreiche Herausbildung und vor allem das Wachstum von Ener- giegenossenschaften voraussetzungsvoll sind. $\mathrm{Zu}$ den Voraussetzungen gehören tragfähige und in Anpassung an wechselnde Rahmenbedingungen weiterzuentwickelnde Geschäftsmodelle sowie zunehmend auch professionalisierte Strukturen, die sich im Wettbewerb mit anderen Anbietern, auch konkurrierenden Energiegenossenschaften, bewähren müssen. Für überregionale und vor allem für deutschlandweit tätige Energiegenossenschaften basieren solche Geschäftsmodelle vor allem auf Größenvorteilen und können nicht vielfach reproduziert werden. Insbesondere die Zahl nationaler Energiegenossenschaften kann nicht beliebig gesteigert werden, sodass ein entsprechender Entwicklungspfad wahrscheinlich nur von wenigen Energiegenossenschaften erfolgreich beschritten werden kann.

Ein wichtiges Ergebnis ist, dass auch regionale Energiegenossenschaften, trotz in der Regel fehlender Größenvorteile im energiebezogenen Kerngeschäft, erfolgversprechende Entwicklungsmöglichkeiten haben, wenn sie ihre Geschäftsmodelle innovativ weiterentwickeln. So deutet das Fallbeispiel der EGO darauf hin, dass die zentrale Herausforderung für regional ausgerichtete Strategien darin liegt, möglichst viele unterschiedliche Akteure vor Ort einzubeziehen und Mitglieder bzw. Kunden über die erfolgreiche Vermittlung eines regionalen Mehrwerts an sich zu binden. Dies kann neben der Diversifizierung innerhalb des Energiebereichs (neben der Strom- bzw. Wärmeproduktion und -vermarktung auch Energieeffizienz- und Einsparcontracting-Dienstleistungen) auch andere Geschäftsfelder der örtlichen Daseinsvorsorge beinhalten, die nur indirekt mit Energiedienstleistungen verbunden sind (z. B. Immobilienbewirtschaftung, Mobilitätsdienstleistungen). Damit sind erfolgreiche regionale Geschäftsmodelle nicht einfach übertragbar, sondern müssen kontextspezifisch angepasst und entwickelt werden. Dennoch sind hier wechselseitige Lernprozesse auf jeden Fall sinnvoll und vor allem möglich, da sie - ähnlich wie übrigens bei den Sparkassen und den Genossenschaftsbanken - in der Regel nicht zu gegenseitigen Beeinträchtigungen führen. Hierbei können professionelle Dach- und andere Verbundorganisationen maßgebliche Funktionen übernehmen und außerdem unterstützend tätig sein, wenn es darum geht, den Ein- und Verkauf sowie das Abrechnungswesen gemeinsam effizienter zu organisieren (Klemisch 2014b: 160).

Während für überregionale Energiegenossenschaften also in erster Linie Größenvorteile (economies of scale) eine wichtige Rolle spielen, sind für regionale Energiegenossenschaften Verbundvorteile (economies of scope) auf der regionalen Ebene besonders bedeutsam und erfordern die sozial innovative „Neukombination lokalen Wissens bzw. lokaler Wissensträger" (Schwarz/Birke/Beerheide 2010: 166). Die Fallbeispiele mit ihren jeweils sehr spezifischen Entwicklungen und Innovationsbiographien weisen außer- 
dem auf Diversifikation als gemeinsamen Erfolgsfaktor hin. Konkret stellt sich in den beiden Beispielen die Direktvermarktung als ein tragendes Element dar, mit dem die Vorteile des genossenschaftlichen Identitätsprinzips und die damit verbundenen Mitbestimmungsrechte realisiert werden können. Hierbei bildet die Bindung von Mitgliedern bzw. Abnehmern der erzeugten Energie eine zentrale Herausforderung. Diese kann auf unterschiedliche Weise gemeistert werden und beinhaltet die Fokussierung auf bestimmte regional bzw. ideologisch ansprechbare Zielgruppen, für die nichtmaterielle Ziele bedeutender sind als eine reine Kosten- bzw. Renditeorientierung. Die dafür erforderliche Überzeugungsarbeit ist eine Vermittlungsaufgabe, die in den Geschäftsmodellen von regionalen ebenso wie von überregionalen Energiegenossenschaften adressiert und verankert werden muss. Zusammenfassend lässt sich festhalten, dass die soziale Innovation Energiegenossenschaft in Zukunft nur dann weiterhin so erfolgreich bleiben kann wie bisher, wenn sie ihre verschiedenen Geschäftsmodelle innovativ weiterentwickelt und sich dabei stärker von den drei genossenschaftlichen Kernprinzipien (vgl. oben Abschnitt 2.1) einschließlich des Identitätsprinzips leiten lässt.

Danksagung Wir danken Susanne Schubert (Darmstadt), Romy Simke, Elena Tillmann und Thomas Meister (alle Bonn) sowie außerdem zwei anonymen Gutachtern für wichtige Hinweise zur Verbesserung des Beitrags.

\section{Literatur}

BBEn - Bündnis Bürgerenergie e. V. (Hrsg.) (2014): Energiewende braucht Bürgerenergie. Vorrang für dezentrale Erzeugung und Direktversorgung mit Bürgerstrom statt Re-Zentralisierung des Energiesektors. Positionspapier des Bündnis Bürgerenergie e.V. zur EEG-Novelle 2014. https://www.buendnis-buergerenergie. de/fileadmin/user_upload/downloads/Positionspapiere/BBEn_ Positionspapier_EEG-Novelle.pdf (14.12.2014).

BDEW - Bundesverband der Energie- und Wasserwirtschaft; EY Ernst \& Young GmbH (Hrsg.) (2015): Gewohnte Wege verlassen. Innovation in der Energiewirtschaft. Stadtwerkestudie Juni 2015. Düsseldorf. http://www.ey.com/Publication/vwLUAssets/ EY-Stadtwerkestudie-2015/\$FILE/EY-Stadtwerkestudie-2015. pdf (11.04.2016).

Berlo, K.; Wagner, O. (2011): Zukunftsperspektiven kommunaler Energiewirtschaft. In: Raumplanung 158/159, 236-242.

Blättel-Mink, B. (2008): Soziologie und Innovation. In: Ebner, A.; Heine, K.; Schnellenbach, J. (Hrsg.): Innovation zwischen Markt und Staat. Die institutionelle Dynamik des wirtschaftlichen Wandels. Baden-Baden, 286-308.

Brücher, W. (2009): Energiegeographie: Wechselwirkungen zwischen Ressourcen, Raum und Politik. Berlin, Stuttgart.

Bruns, E.; Köppel, J.; Ohlhorst, D.; Schön, S. (2008): Die Innovationsbiographie der Windenergie. Absichten und Wirkungen von Steuerungsimpulsen. Berlin, Münster. = Innovationsforschung, 2.

Bruns, E.; Ohlhorst, D.; Wenzel, B.; Köppel, J. (2011): Renewable Energies in Germany's Electricity Market. A Biography of the Innovation Process. Dordrecht, Heidelberg, London, New York.

Cowell, R.; Bristowa, G.; Munday, M. (2011): Acceptance, acceptability and environmental justice: the role of community benefits in wind energy development. In: Journal of Environmental Planning and Management 54, 4, 539-557.

Degenhart, H.; Nestle, U. (2014): Marktrealität von Bürgerenergie und mögliche Auswirkungen von regulatorischen Eingriffen. Eine Studie für das Bündnis Bürgerenergie e.V. (BBEn) und den Bund für Umwelt und Naturschutz Deutschland e.V. (BUND). Lüneburg, Kiel. http://www.bund.net/fileadmin/ bundnet/pdfs/klima_und_energie/140407_bund_klima_energie_ buergerenergie_studie.pdf (26.02.2015).

DGRV - Deutscher Genossenschafts- und Raiffeisenverband e.V. (Hrsg.) (2012): Energiegenossenschaften. Ergebnisse der Umfrage des DGRV und seiner Mitgliedsverbände im Frühsommer 2012. Berlin.

DGRV - Deutscher Genossenschafts- und Raiffeisenverband e.V. (Hrsg.) (2013): Energiegenossenschaften. Ergebnisse der Umfrage des DGRV und seiner Mitgliedsverbände. Frühjahr 2013. Berlin.

DGRV - Deutscher Genossenschafts- und Raiffeisenverband e.V. (Hrsg.) (2014): Energiegenossenschaften. Ergebnisse der Umfrage des DGRV und seiner Mitgliedsverbände. Frühjahr 2014. Berlin.

EGO - Energiegenossenschaft Odenwald e.G. (Hrsg.) (2014a): Jahresbericht 2013. Erbach.http://www.energiegenossenschaftodenwald.de/index.php/mitglied-werden/mitgliedschaft/ geschaeftsbericht/download/5-geschaeftsberichte/1geschaeftsbericht-2013 (06.03.2015).

EGO - Energiegenossenschaft Odenwald e. G. (Hrsg.) (2014b): Zukunft gemeinsam gestalten. Ökonomisch, ökologisch, sozial. Erbach. http://www.energiegenossenschaft-odenwald.de/index.php/ downloads/send/7-broschuere/21-ego-imagebroschuere (27.04.2016).

EGO - Energiegenossenschaft Odenwald e.G. (Hrsg.) (o. J.): Aktuelles zum Thema Windkraft im Odenwaldkreis. http://www. energiegenossenschaft-odenwald.de/index.php/hauptmenutest/ 58-2014/254-aktuelles-zum-thema-windkraft-im-odenwaldkreis2 (27.04.2016).

Elsen, S. (2012): Genossenschaften als Organisationen der sozialen Innovation und nachhaltigen Entwicklung. In: Beck, G.; Kropp, C. (Hrsg.): Gesellschaft innovativ: Wer sind die Akteure?. Wiesbaden, 85-102.

EnGeno - Carl Von Ossietzky Universität Oldenburg; HelmholtzZentrum für Umweltforschung; IdE Institut Dezentrale Energietechnologien (Hrsg.) (2014): Positionspapier. Bürgerbeteiligung - Energiewende - Dezentralität? Kernziele der Energiewende in Gefahr!. Ein Positionspapier des Forschungsprojektes EnGeno zur aktuellen energiepolitischen Entwicklung (Langfassung). Oldenburg. http://engeno.net/wp-content/uploads/2013/ 09/EnGeno_Positionspapier_Langfassung.pdf (05.04.2016).

Engerer, H. (2014): Energiegenossenschaften in der Energiewende. Berlin. http://www.diw.de/documents/publikationen/73/diw 01.c.470187.de/diw_roundup_30_de.pdf (05.04.2016). = DIW Roundup, 30.

Fischer, B.; Moser, P.; Schenk, K. (2015): Zukunftsfelder der Energieversorgung: Entwicklungsoptionen, Interessenlagen und Strukturen in drei erneuerbaren Entwicklungspfaden. http://engeno.net/ wp-content/uploads/2013/09/09_02_2015_Zukunftsfelder-derEnergieversorgung_final.pdf (09.03.2015).

Flieger, B. (2010): Energiegenossenschaft Odenwald eG. Ausgeprägte Kooperation verschiedener Akteure als Erfolgsfaktor. In: Contraste $27,306,8$.

Flieger, B. (2011): Photovoltaikgenossenschaften. Lokale Wertschöpfung durch Bürgerbeteiligung. In: Verbands-Management. Fachzeitschrift für Verbands- und Nonprofit-Management 37, 1, $50-57$.

George, W. (2012): Vorteile von Genossenschaftslösungen in der Energiewende. In: Informationen zur Raumentwicklung 9-10/2012, 503-513. 
Greenpeace Energy eG (Hrsg.) (2014a): Genossenschaft. Vertrauen Sie keinem Energieversorger, der Ihnen nicht gehört. http://www. greenpeace-energy.de/fileadmin/docs/broschueren/greenpeace_ energy_genossenschaft_broschuere.pdf (09.03.2015).

Greenpeace Energy eG (Hrsg.) (2014b): Geschäftsbericht 2013. https://mitglieder.greenpeace-energy.de/fileadmin/docsgenoportal/geschaeftsberichte/Greenpeace_Energy_Gesch \%C3\%A4ftsbericht_2013.pdf (27.04.2016).

Henkel, A. (2013): Energiegenossenschaften: Avantgarde des ökonomischen Wandels?. Masterarbeit, Leuphana Universität Lüneburg.

Hirschl, B.; Aretz, A.; Prahl, A.; Böther, T.; Heinbach, K.; Pick, D.; Funcke, S. (2010): Kommunale Wertschöpfung durch Erneuerbare Energien. Studie im Auftrag der Agentur für Erneuerbare Energien. Berlin. $=$ Schriftenreihe des IÖW, 196/10.

Holstenkamp, L.; Degenhart, H. (2013): Bürgerbeteiligungsmodelle für erneuerbare Energien. Eine Begriffsbestimmung aus finanzwirtschaftlicher Perspektive. Lüneburg. http://www.leuphana.de/ fileadmin/user_upload/PERSONALPAGES/_ijkl/janner_steve/ Homepage_Master/wpbl_13.pdf (30.03.2014). = Working Paper Series in Business and Law, 13.

Holstenkamp, L.; Müller, J. R. (2013): Zum Stand von Energiegenossenschaften in Deutschland. Ein statistischer Überblick zum 31.12.2012. Lüneburg. http://www.leuphana.de/fileadmin/ user_upload/PERSONALPAGES/_ijkl/janner_steve/Homepage_ Master/wpbl_14.pdf (18.03.2015). = Working Paper Series in Business and Law, 14.

Howaldt, J.; Schwarz, M. (2010): Soziale Innovation - Konzepte, Forschungsfelder und -perspektiven. In: Howaldt, J.; Jacobsen, H. (Hrsg.): Soziale Innovation. Auf dem Weg zu einem postindustriellen Innovationsparadigma. Wiesbaden, 87-108.

ICA (International Co-operative Alliance) (2011): What is a co-operative?. http://ica.coop/en/what-co-operative (30.03.2014).

Itten, A.; Mono, R. (2014): Wie Bürger die Energiewende mitgestalten. In: Ökologisches Wirtschaften 29, 1, 20-21.

Jakubowski, P.; Koch, A. (2012): Energiewende, Bürgerinvestition und regionale Entwicklung. In: Informationen zur Raumentwicklung, 9-10/2012, 475-490.

Klagge, B.; Brocke, T. (2012): Decentralized electricity generation from renewable sources as a chance for local economic development: a qualitative study of two pioneer regions in Germany. In: Energy, Sustainability and Society 2, 5, 1-9. http://www.springeropen.com/track/pdf/10.1186/2192-0567-25 ?site=energsustainsoc.springeropen.com (18.03.2015).

Klagge, B.; Brocke, T. (2013): Energiewende vor Ort: Dezentrale Stromerzeugung und die Rolle von Stadtwerken und Regionalversorgern. In: Geographische Rundschau 65, 1, 12-18.

Klemisch, H. (2014a): Die Rolle von Genossenschaften in der Energiewende. In: Ökologisches Wirtschaften 29, 1, 22-23.

Klemisch, H. (2014b): Energiegenossenschaften als regionale Antwort auf den Klimawandel. In: Schröder, C.; Walk, H. (Hrsg.): Genossenschaften und Klimaschutz. Akteure für zukunftsfähige, solidarische Städte. Wiesbaden, 149-166.

Lasch, H. (2009): Der Wind bezahlt den Kindergarten. In: Links, V.; Volke, K. (Hrsg.): Zukunft erfinden. Kreative Projekte in Ostdeutschland. Berlin, 16-20.

Li, W. L.; Birmele, J.; Schaich, H.; Konold, W. (2013): Transitioning to community-owned renewable energy: Lessons from Germany. In: Procedia Environmental Sciences, 17, 719-728.

Maly, C.; Meister, M.; Schomerus, T. (2014): EEG 2014 - Das Ende der Bürgerenergie?. In: Energierecht. Zeitschrift für die gesamte Energierechtspraxis 3, 4, 147-154.

Maron, B.; Maron, H. (2012): Genossenschaftliche Unterstützungsstrukturen für eine sozialräumlich orientierte Energiewirtschaft. Köln. = KNI PAPERS, 01/2012.

Masson, T.; Centgraf, S.; Rauschmayer, F.; Simke, R. (2015): Mitglieder-Zuwachspotenzial für Energiegenossenschaften in Deutsch- land. In: Zeitschrift für das gesamte Genossenschaftswesen 65 , 3, 191-208.

Müller, J. R.; Holstenkamp, L. (2015): Zum Stand von Energiegenossenschaften in Deutschland. Aktualisierter Überblick über Zahlen und Entwicklungen zum 31.12.2014. http://www. leuphana.de/fileadmin/user_upload/Forschungseinrichtungen/ professuren/finanzierung-finanzwirtschaft/files/Arbeitspapiere/ wpbl20_energiegenossenschaften2014_final.pdf (26.02.2015). = Working Paper Series in Business and Law, 20.

Netzwerk Energiewende jetzt e. V. (Hrsg.) (2016): Energiegenossenschaften: Neugründungen 2015 auf niedrigem Niveau. Meldung vom 21. März 2016. http://www. energiegenossenschaften-gruenden.de/news.html?\& cHash=73eb8f00bb9f6b4c53c61d54fc9a3ffc\&tx_ttnews $\% 5 \mathrm{Bttnews} \% 5 \mathrm{D}=291$ (18.04.2016).

Ohlhorst, D.; Schön, S. (2015): Constellation Analysis as a Means of interdisciplinary Innovation Research - Theory formation from the bottom up. In: Historical Social Research/Historische Sozialforschung 40, 3, 258-278.

Radtke, J. (2013): Bürgerenergie in Deutschland - ein Modell für Partizipation?. In: Radtke, J. (Hrsg): Die deutsche „Energiewende" nach Fukushima. Der wissenschaftliche Diskurs zwischen Atomausstieg und Wachstumsdebatte. Marburg, 119-138.

Radtke, J. (2014): Die Energiewende in Deutschland und die Partizipation der Bürger. Berlin. http://www.b-b-e.de/fileadmin/inhalte/ aktuelles/2014/02/NL02_Gastbeitrag_Radtke.pdf (10.11.2014). = BBE-Newsletter, 2014/02.

Rammert, W. (2000): National Systems of Innovation, Idea Innovation Networks, and Comparative Innovation Biographies. Some Comments on Jerald Hage's and Rogers Hollingsworth's Considerations about „The Role of Institutions and Organizations in the Innovation Process“. Berlin, 35-42. = Technical University Technology Studies Working Paper, TUTS-WP-5-2000.

Ribi, F.; Buser, B.; von Felten, N.; Walther, R.; Bernath, K. (2012): Regionalökonomische Potenziale und Erfolgsfaktoren für den Aufbau und Betrieb von Energieregionen. http://www.energie-region. ch/fileadmin/user_upload/Energieregion/de/Dateien/Dokumente/ Wirtschaftspotenziale_Energieregionen.pdf (31.05.2016).

Schmole, H. (2014): Energiegenossenschaften in Deutschland - eine Analyse der Geschäftsmodelle und ihrer Übertragbarkeit auf Österreich und die Schweiz. Masterarbeit, Universität Bonn.

Schreuer, A.; Weismeier-Sammer, D. (2010): Energy cooperatives and local ownership in the field of renewable energy technologies: A literature review. Vienna. = RICC Research Report, 2010/4.

Schröder, C.; Walk, H. (2013): Local Climate Governance and the Role of Cooperatives. In: Knieling, J.; Filho, W. L. (Hrsg.): Climate Change Governance. Heidelberg, 105-118.

Schröder, C.; Walk, H. (Hrsg.) (2014): Genossenschaften und Klimaschutz. Akteure für zukunftsfähige, solidarische Städte. Wiesbaden.

Schwarz, J.; Birke, M.; Beerheide, E. (2010): Die Bedeutung sozialer Innovation für eine nachhaltige Entwicklung. In: Howaldt, J.; Jacobsen, H. (Hrsg.): Soziale Innovation. Auf dem Weg zu einem postindustriellen Innovationsparadigma. Wiesbaden, 165-180.

Staab, J. (2013): Erneuerbare Energien in Kommunen. Energiegenossenschaften gründen, führen und beraten. Heidelberg.

Thomi, W.; Werner, R. (2001): Regionale Innovationssysteme. Zur territorialen Dimension von Wissen und Innovation. In: Zeitschrift für Wirtschaftsgeographie 45, 3-4, 202-218.

Trend:research; Leuphana Universität Lüneburg (2013): Definition und Marktanalyse von Bürgerenergie in Deutschland. Bremen, Lüneburg. https://www.buendnis-buergerenergie.de/fileadmin/user_ upload/downloads/Studien/Studie_Definition_und_Marktanalyse_ von_Buergerenergie_in_Deutschland_BBEn.pdf (25.03.2014).

Trend:research; KNI - Klaus Novy Institut e.V. (2011): Marktakteure. Erneuerbare-Energien-Anlagen in der Stromerzeugung. Köln. http://www.kni.de/media/pdf/Marktakteure_Erneuerbare_ Energie_Anlagen_in_der_Stromerzeugung_2011.pdf (11.04.2016). 
Viardot, E. (2013): The role of cooperatives in overcoming the barriers to adoption of renewable energy. In: Energy Policy 63, 756-764.

Volz, R. (2011): Zur Umsetzung des Förderauftrags in Energiegenossenschaften. In: Zeitschrift für das gesamte Genossenschaftswesen 61, 4, 289-304.

Volz, R. (2012a): Bedeutung und Potenziale von Energiegenossenschaften in Deutschland. Eine empirische Aufbereitung. In: Informationen zur Raumentwicklung 9-10/2012, 515-524.

Volz, R. (2012b): Genossenschaften im Bereich erneuerbarer Energien: Status quo und Entwicklungsmöglichkeiten eines neuen Betätigungsfeldes. Stuttgart-Hohenheim.
Walker, G. (2008): What are the barriers and incentives for communityowned means of energy production and use?. In: Energy Policy $36,12,4401-4405$.

Yildiz, Ö.; Rommel, J.; Debor, S.; Holstenkamp, L.; Mey, F.; Müller, J. R., Radtke, J.; Rognli, J. (2015): Renewable energy cooperatives as gatekeepers or facilitators? Recent developments in Germany and a multidisciplinary research agenda. In: Energy Research \& Social Science 6, 59-73.

Zoellner, J.; Rau, I.; Schweizer-Ries, P. (2011): Beteiligungsprozesse und Entwicklungschancen für Kommunen und Regionen. In: Ökologisches Wirtschaften 26, 3, 25-27. 